\title{
Anterior Cingulate Neurons Represent Errors and Preparatory Attention within the Same Behavioral Sequence
}

\author{
Nelson K. B. Totah, ${ }^{1,2,3}$ Yun Bok Kim, ${ }^{1}$ Houman Homayoun, ${ }^{1}$ and Bita Moghaddam ${ }^{1,2}$ \\ ${ }^{1}$ Department of Neuroscience, University of Pittsburgh, ${ }^{2}$ Center for Neuroscience at the University of Pittsburgh, and ${ }^{3}$ Center for the Neural Basis of \\ Cognition, Pittsburgh, Pennsylvania 15260
}

The anterior cingulate cortex (ACC) has been implicated in both preparatory attention (i.e., selecting behaviorally relevant stimuli) and in detecting errors. We recorded from the rat ACC and medial prefrontal cortex (mPFC), which is functionally homologous to the primate dorsolateral PFC, during an attention task. The three-choice serial reaction time task requires a rat to orient toward and divide attention between three brief ( $300 \mathrm{~ms}$ duration) light stimuli presented in random order across nose poke holes in an operant chamber. In both the ACC and mPFC, we found that neural activity was related to the level of preparatory (precue) attention and subsequent correct or incorrect choice, in that the magnitude of the single units' response to the cue was lower on incorrect trials and was not different than baseline on unattended trials. This preparatory neural activity consisted of both excitatory and inhibitory phasic responses. The number of units responding to the cue was similarly graded, in that fewer units exhibited phasic responses to the cue on incorrect and unattended trials, compared with correct trials. Although preparatory activity was found in both the ACC and mPFC, activity after incorrect nose pokes, which may be related to error detection, were only observed in the ACC. Thus, during the same behavioral sequence, the ACC encodes both error-related events and preparatory attention, whereas the $\mathrm{MPFC}$ only participates in preparatory attention. The finding of substantial inhibitory activity during the preparatory period suggests a critical role for inhibition of pyramidal cells in PFC-mediated cognitive functions.

\section{Introduction}

Proper goal-directed behavior requires that an organism internalizes behaviorally relevant stimuli, brings appropriate stimulus-response rules online, and monitors outcomes to adjust behavior accordingly. Preparatory attention or the selection of taskrelevant stimuli is critical for the execution of proper goaldirected behavior. The medial and dorsolateral prefrontal cortices (mPFC/DLPFC) and the anterior cingulate cortex (ACC) have both been implicated in preparatory attention (Miller and Cohen, 2001; Botvinick et al., 2004). Views on the role of the ACC, however, differ. A leading theory suggests this region detects errors and then signals the DLPFC to adjust the level of preparatory attention (Botvinick et al., 2004). This view holds that ACC neurons should selectively respond to instances of error detection, which potentially represent a negative reward prediction error signal (Nieuwenhuis et al., 2004) or uncertainty, but ACC neurons should not necessarily respond during preparatory attention to upcoming goal-relevant stimuli. Another theory suggests that the ACC is involved in preparatory attention (Posner and DiGirolamo, 1998; Weissman et al., 2005; Roelofs et al.,

Received March 9, 2009; revised March 30, 2009; accepted April 1, 2009.

This work was supported by the National Institute of Mental Health, a National Research Service Award institutional training grant, and the Center for the Neural Basis of Cognition. We thank Dr. Rob Kass (Carnegie Mellon University, Pittsburgh, PA) for consultation on the statistical methods used herein and Dr. Charles Bradberry (University of Pittsburgh) for helpful discussions.

Correspondence should be addressed to Bita Moghaddam, Department of Neuroscience, University of Pittsburgh, A210 Langley Hall, Pittsburgh, PA 15260. E-mail: bita@pitt.edu.

D01:10.1523/JNEUROSCI.1142-09.2009

Copyright $\odot 2009$ Society for Neuroscience $\quad$ 0270-6474/09/296418-09\$15.00/0
2006). This is supported by the observation of attentional impairments after lesions of the rat ACC (Passetti et al., 2002; Chudasama et al., 2003; Ng et al., 2007) and by primate single-unit recordings demonstrating excitatory phasic activity during prestimulus periods (Shidara and Richmond, 2002; Roelofs et al., 2006; Johnston et al., 2007).

To elucidate the neurophysiological and anatomical basis of preparatory attention, we conducted single-unit recordings in the rat ACC and prelimbic cortex (mPFC) during an attention task. The rat $\mathrm{mPFC}$ and ACC are analogous to the human and primate $\mathrm{mPFC} / \mathrm{DLPFC}$ and ACC, respectively, although this homology is mainly based on functionality (Uylings et al., 2003). During the task, rats were required to wait on a brief stimulus and then respond quickly. Stimulus and response conflict were not used; thus, we assumed that precue neuronal activity reflected preparatory attention to select the upcoming stimuli, rather than conflict resolution. Another feature of the task was that the rat had the opportunity to make incorrect response choices. Thus, we could test if detection of errors and upcoming goal-relevant stimuli during a single trial are represented differently in the ACC versus the $\mathrm{mPFC}$.

We found that error-related phasic responses were selective for the ACC, whereas both regions exhibited preparatory activity. This suggests a dual role for the ACC; in addition to signaling error-related events, it is involved in selection of goal-relevant stimuli along with the mPFC. Furthermore, the preparatory phasic responses were both inhibitory and excitatory, suggesting that different afferent regulation of putative PFC pyramidal neurons is important for preparatory attention. The inhibition of PFC 


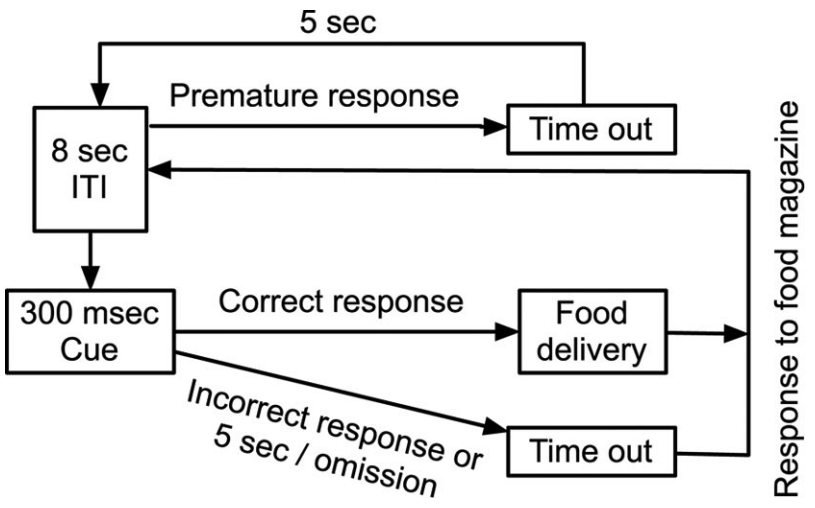

Figure 1. The three-choice serial reaction time task. A recording session lasted $30 \mathrm{~min}$. After an $8 \mathrm{~s}$ ITI, one of three cue locations was randomly illuminated for $300 \mathrm{~ms}$. If the rat made a premature cue hole nose poke before cue illumination, the house light was extinguished for $5 \mathrm{~s}$ and then a new trial was started. The rat had $5 \mathrm{~s}$ to respond into one of the cue holes. A correct response led to reward delivery into the food magazine on the wall opposite from the cue holes. An incorrect response or omission of response led to house light extinguishment. After an error, a new trial was begun, and the house light was illuminated again after the rat nose poked into the empty food magazine.

pyramidal units during attention may be attributable to excitation of PFC interneurons via thalamic inputs (Rotaru et al., 2005). The phasic inhibition is also consistent with the well established role of neuromodulators, such as dopamine and norepinephrine in modulating PFC function in that the primary postsynaptic effect of these neurotransmitters on PFC pyramidal neurons is inhibition (Mantz et al., 1988; Pirot et al., 1992; Lewis and O'Donnell, 2000).

\section{Materials and Methods}

Animals. Male Sprague Dawley rats were used. Twenty-four rats were used for the task characterization experiments and 10 were used for the electrophysiology experiments. All rats were housed on a reverse light cycle and trained and tested in dark red light during the rats' active phase (7:00 A.M.-7:00 P.M.). Both task characterization and recording studies used two different cohorts of rats for each study. All experiments were performed in compliance with the University of Pittsburgh Institutional Care and Use Committee.

Behavioral task. The task is based on the five-choice serial reaction time task (Carli et al., 1983), except that three-choice operant chambers were used to accommodate the existing behavioral electrophysiology setup. Experiments were performed in operant chambers with a house light on the ceiling, three cue holes with internal light-emitting diodes on one wall, and an illuminated food magazine on the wall opposite the cue holes. Nose pokes into the cue holes and food magazine were registered by photosensors. Training began after 1 week of handling and chamber habituation. A correct response, consisting of a nose poke into a lit cue, was rewarded with two sucrose pellets. Incorrect responses into an unlit cue or not responding within $5 \mathrm{~s}$ after stimulus presentation (an omission) were punished with a house-light off "timeout." The rat initiated the next trial with a poke into the empty food magazine, thus allowing the rat to reduce the duration of the timeout. An $8 \mathrm{~s}$ intertrial interval (ITI) passed before the next cue presentation. Premature responses during the ITI were repeatedly punished with $5 \mathrm{~s}$ timeouts before restarting the ITI. See Figure 1 for the task layout. Rats were trained in six successive "levels" which used reduced-cue durations at each level $(15,5,2,1 \mathrm{~s}, 500$ and 300 $\mathrm{ms})$. Each individual rat progressed through training levels after meeting performance criteria. The first four training levels used the following criteria: $>80 \%$ accuracy and $<20 \%$ omissions for three consecutive sessions. At the $500 \mathrm{~ms}$ level, rats had to perform at $>75 \%$ accuracy for six consecutive sessions and $<20 \%$ omissions. Rats were deemed ready for testing (or surgical implantation) at the $300 \mathrm{~ms}$ level once they met performance criteria of $>70 \%$ accuracy and $<20 \%$ omissions for six consecutive sessions. Sessions consisted of either 30 min or 150 rewards, whichever occurred first. At the $300 \mathrm{~ms}$ cue duration, the session lasted 30 min regardless of reward obtained. Percentage accuracy [ $100^{\star}$ (\# of correct responses/\# of total responses)] was taken as the measure of attentional performance. Rats exhibited attentive behavior, whereby they oriented to the cues and waited for the signal; it was extremely rare (approximately $<1 \%$ of trials across many 30 min sessions) for rats to orient away from the cues once they had begun orienting to them.

Electrophysiology procedure. On completion of training, rats $(n=10)$ were implanted under isoflurane anesthesia with two microelectrode arrays each consisting of eight Teflon-insulated stainless steel wires in a $2 \times 4$ pattern measuring $0.25 \mathrm{~mm}$ by $0.70 \mathrm{~mm}$ with an impedance of $300-700 \mathrm{k} \Omega$ (NB Labs). One array was placed spanning the ACC: -0.2 to $-1.0 \mathrm{~mm}$ anterior to bregma, -0.4 to $-0.7 \mathrm{~mm}$ lateral to bregma, and $-2.5 \mathrm{~mm}$ ventral from the dura surface, and one array was placed spanning the contralateral mPFC (prelimbic cortex): +2.4 to $+3.4 \mathrm{~mm}$ anterior to bregma, +0.5 to $+0.8 \mathrm{~mm}$ lateral to bregma, and $-3.8 \mathrm{~mm}$ ventral from the dura surface.

After 1 week of recovery, rats were acclimated to attachment of the electrode cable in the operant box over $4 \mathrm{~d}$. Single units were recorded via a unity-gain field-effect transistor headstage and lightweight cabling which passed through a commutator to allow freedom of movement. Signals were amplified using a $1000 \times$ gain and a $220-5900 \mathrm{~Hz}$ bandpass filter and were digitized at a rate of $40 \mathrm{kHz}$. Recorder software (Plexon) was used to record waveforms crossing a channel-specific voltage threshold, and signals from the operant box were used as event markers to coordinate behavioral events with the signal from the microelectrode array. Units were isolated in off-line sorter software (Plexon) using a three-dimensional space generated from the first three principle components of the data, as well as two-dimensional spaces generated from peak and valley amplitudes. Stability of units over time was confirmed by viewing the waveform clusters in a three-dimensional space of the first two principle components and time. Units were rejected if the interspike interval histogram was inconsistent with a refractory period of $<1.1 \mathrm{~ms}$. In general, 2-3 units were clearly isolated per electrode. In 10 rats, the mean number of units recorded in the ACC was 10.6, and the range was 20. In the mPFC, the mean number of units recorded was 15.3, and the range was 25. Supplemental Figure S1, available at www.jneurosci.org as supplemental material, shows an example waveform cluster of three isolated single units.

Neural activity was recorded during three $30 \mathrm{~min}$ sessions using the $300 \mathrm{~ms}$ cue duration, and the session with the best behavioral performance was chosen for analysis. An 8 s ITI was used in the recording sessions to provide sufficient time for the rat to retrieve reward from the food magazine, initiate the next trial, and turn to orient to the cues. Sessions were videotaped and reviewed to eliminate trials on which the rat faced a side wall because those were not stereotypical task behaviors (see supplemental Table 1, available at www.jneurosci.org as supplemental material, for frequency of these trials). Video analysis also demonstrated that grooming occurred rarely and thus did not affect single-unit activity (supplemental Table S1, available at www.jneurosci.org as supplemental material). Review of task performance on video was used to select omission trials during which the rat was oriented away from the cues and not behaviorally engaged in waiting for the brief cue illumination.

Analysis of electrophysiological data. Peri-event firing rate time histograms (PETH) were used to analyze neuronal responses to behavioral events (cue onsets; correct, incorrect, and premature cue hole nose pokes; and food magazine nose pokes). Neural activity was analyzed in peri-event windows with the behavioral event of interest at time $=0$. Windows were chosen that correlated with the behavior of interest. A -1 to $+1 \mathrm{~s}$ window was used for analysis of all behavioral events, except the precue window, which was -4 to $0 \mathrm{~s}$ in relation to cue onset. The larger precue window was chosen because, on average, the rats began facing the cues and remained in an oriented posture starting 2-3 s before cue onset. Details of orientation times are provided in supplemental Table S1, available at www.jneurosci.org as supplemental material. Precue neural activity was compared between the three trials types, determined by the sub- 
sequent cue hole nose poke response (or lack of nose poke response) after the cue onset. Phasic changes in firing rate were measured by calculating a $z$-score using the firing rate during -6 to $-4 \mathrm{~s}$ before cue onset; the baseline was calculated on a cell-by-cell basis.

Analysis of electrophysiological data was performed in Matlab. The $z$-score was calculated from an approximate Poisson distribution of the expected firing rate, where $Z=(($ observed $\#$ of spikes per bin) - (expected \# of spikes))/ ( $\checkmark$ expected \# of spikes) and the expected \# of spikes $=(($ total $\#$ of spikes during baseline time period $) /($ baseline time duration $)) *($ time bin size). A $z$-score $\geq 2.36$ for $x$ consecutive time bins was used to determine a unit's significant $(p<0.01)$ phasic response to a behavioral event. For correct, incorrect, and premature cue hole nose poke events and for food magazine nose pokes, $x=4$, and a $50 \mathrm{~ms}$ time bin was used for analysis in small peri-event time windows. For precue activity, $x=3$, and a $200 \mathrm{~ms}$ bin was used; the larger bin size was used to reduce variability over the larger peri-event time window. To assess if bin size affected our results, we also report the precue neural activity with $x=4$, and a $50 \mathrm{~ms}$ bin in the supplemental data, available at www.jneurosci.org as supplemental material. Population activity was constructed by averaging the firing rate of all significantly responding units. A two-way repeatedmeasures ANOVA was performed with time (repeated measure) and trial type or brain region as main factors to assess differences in population activity.

Histology. At the completion of recordings, rats were anesthetized with chloral hydrate, a $30 \mu \mathrm{A}$ current was passed through the recording array for $10 \mathrm{~s}$, and the rats were perfused with normal saline for $10 \mathrm{~min}$ and $10 \%$ buffered formalin for $10 \mathrm{~min}$. After fixation, brains were sectioned and stained using cresyl violet. Placement of electrode tips was confirmed under a light microscope and single units recorded from improperly placed electrodes were excluded from analysis (Fig. 2).

\section{Results}

\section{Sustained attention task acquisition and performance}

For the task characterization study ( $N=48$ from two equally sized cohorts), $96 \%$ of rats reached criterion performance at the testing level (300 ms cue duration) after 41 training sessions. Accuracy was reduced as cue duration decreased, and at the 300 ms cue duration, accuracy reached $78.8 \pm 0.6 \%$ (Fig. 3 ). Furthermore, all behavioral measures (only accuracy shown) remained stable after training $(p>0.05)$, which indicates that the task is well learned. Omissions reached $5.9 \pm 0.5 \%$, and the number of premature responses reached $16.5 \pm 1.6$. Performance at each task level was calculated from the last three sessions of each cue duration level. Similar levels of performance were observed during the electrophysiology recording sessions (Fig. 4), with slight decreases in performance being attributable to behaving with a recording cable attached.

\section{Precue activity related to attentional control}

A total of 106 units in the ACC and 153 units in the mPFC were recorded from 10 animals. Significant excitatory or inhibitory phasic changes in firing rate were observed before the cue. These responses were divided into three groups depending on whether a correct response, an incorrect response, or an omission of response followed.

Cue-related phasic responses were observed on correct and incorrect trials, but the response magnitude was reduced on incorrect trials. Figure 5 shows example peri-event time histograms of single units with significant precue responses on correct trials and the activity of the same unit on incorrect and omission trials. Although cue-related responses were observed in both the ACC and $\mathrm{mPFC}$, more units in the ACC were responsive before the cue. In the ACC, 27 (25.4\%) out of the total number of recorded units were cue responsive on correct trials, whereas in $\mathrm{mPFC}$ cortex, only 19 (12.4\%) were cue responsive on correct trials (Fig. $5 B, E)$. In both brain regions, the number of cue responsive units was different depending on response outcome, with fewer units phasically responding with excitation or inhibition before the cue on incorrect and omission trials. Table 1 lists the number and percentage of units that responded during the precue period on each trial type. Notably, inhibitory phasic responses to the cue were observed mostly on trials where a subsequent correct response was made, whereas fewer inhibitory responses were observed on incorrect and omission trials.

Figure 5, $C$ and $F$, shows the population activity of single units responsive on correct trials and the activity of those same units on incorrect and omission trials. Similar to the number of cue responsive units, the population response patterns were graded in that the magnitude of response to the cue was lower on incorrect trials and was not different from baseline on omission trials. For excitatory units (solid lines), there was a significant interaction between trial type (correct, incorrect, and omission) and time in both ACC $\left(F_{(58,1305)}=6.76, p<0.0001\right)$ and $\operatorname{mPFC}\left(F_{(58,696)}=\right.$ $1.77, p<0.001)$. A significant interaction between trial type (correct, incorrect, and omission) and time was also observed for inhibitory units (dashed lines) in both $\operatorname{ACC}\left(F_{(58,870)}=3.69, p<\right.$ $0.0001)$ and $\operatorname{mPFC}\left(F_{(58,870)}=3.87, p<0.0001\right)$. When only correct and incorrect trial types were compared for units responding with excitation, there were significant differences in the magnitude of the $z$-score in the ACC (trial type: $F_{(1,30)}=5.98, p<$ 

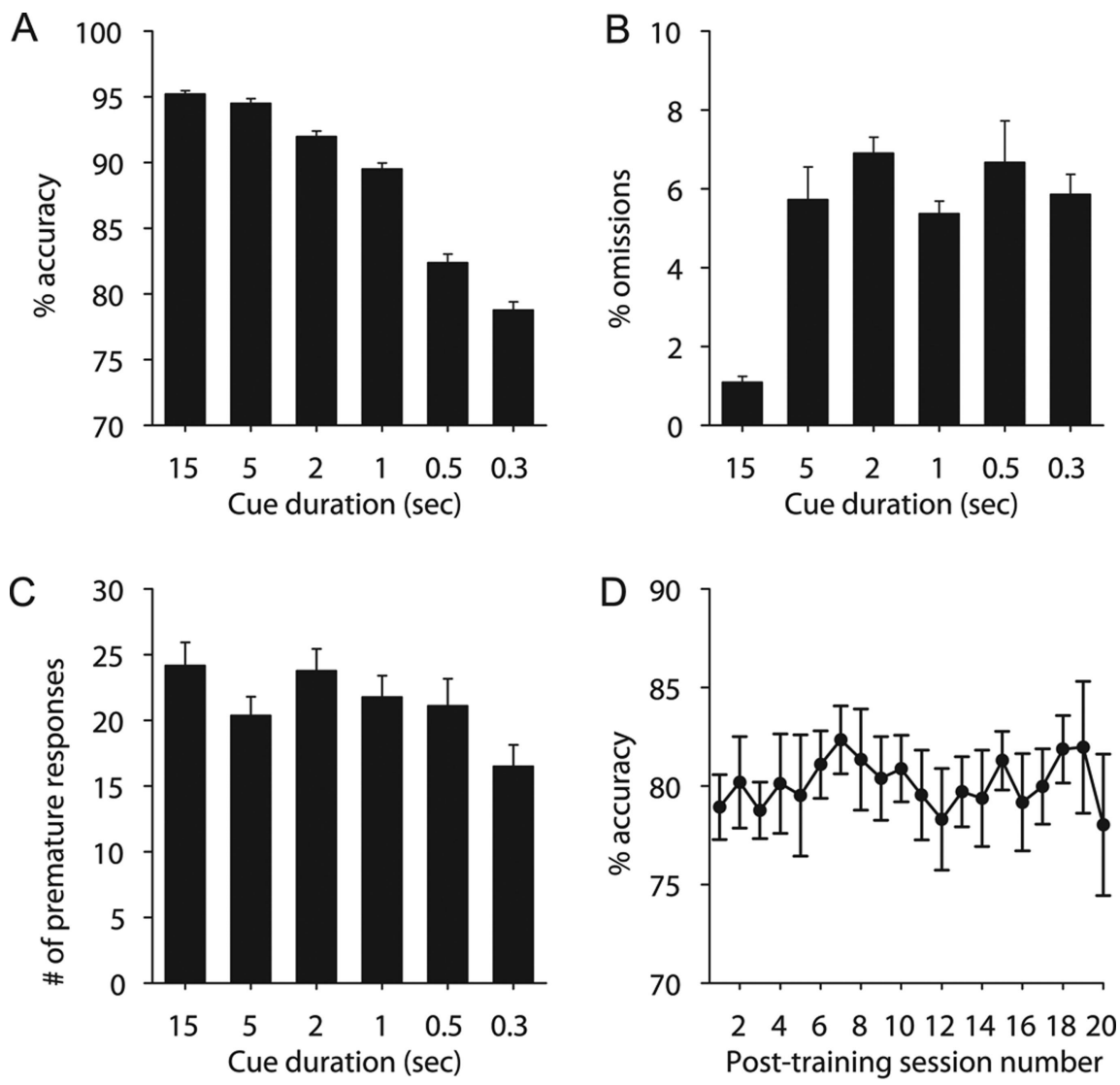

Figure 3. Behavioral task characterization. Rats were trained in the task using an initial cue duration of $15 \mathrm{~s}$. Cue duration was shortened at each training level until the rat met performance criteria using a 300 ms cue duration. Performance criteria were used to move the rat to the next level (see Materials and Methods). For $\boldsymbol{A}-\boldsymbol{C}$, the mean and SE were calculated from the final three sessions of each training level. $\boldsymbol{A}$, Percentage accuracy, the measure of attention, at each training level. Accuracy decreased with shortening cue duration. $\boldsymbol{B}$, Percentage omissions at each training level. $\boldsymbol{C}$, The number of premature responses at each training level. $\boldsymbol{D}$, Accuracy for 20 sessions (300 ms cue duration) after rats met performance criteria at the $300 \mathrm{~ms}$ cue duration. Error bars indicate SEM.

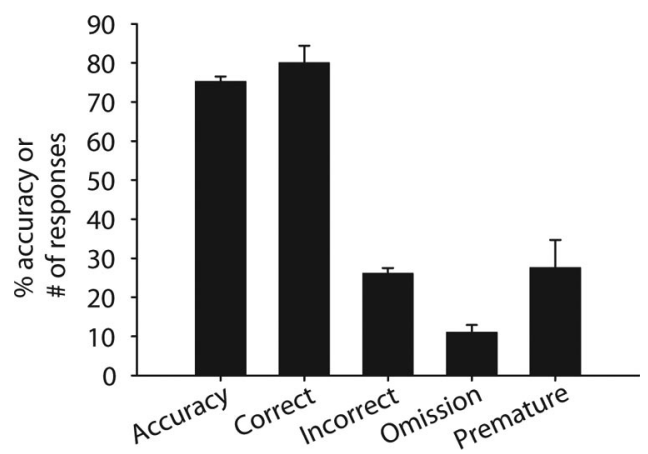

Figure 4. Behavioral task performance for the 10 rats used for electrophysiology. Accuracy refers to the percentage of accuracy, and correct, incorrect, omission, and premature refer to the number of responses for each trial type. Error bars indicate SEM.

0.05 ; time, $F_{(29,870)}=8.22, p<0.0001$; trial $\times$ time interaction: $p>0.05$ ) but not in the mPFC (trial type: $p>0.05$; time: $F_{(29,464)}$ $=4.03, p<0.0001$; trial $\times$ time interaction: $p>0.05)$. However, when correct and incorrect trial types were compared for units responding with inhibition, there was a trend for a trial type and time interaction in the $\operatorname{ACC}\left(F_{(29,580)}=1.43, p=0.067\right)$ and a significant interaction in the $\mathrm{mPFC}$ (trial $\times$ time interaction: $\left.F_{(29,580)}=1.89, p<0.01\right)$. Although the interactions between trial type and time for correct and incorrect trials are not consis- tently significant, the results clearly support the finding of preparatory activity on trials with attention engaged (correct and incorrect trials) compared with unattended trials (omission trials). Review of behavior videos demonstrated that the preparatory behavior was similar for both correct and incorrect trials. Although the latency to respond to the cue nose poke hole is longer for incorrect trials (supplemental Table S1, available at www. jneurosci.org as supplemental material), this is likely a correlate of increased decision-making time. The activity ( $z$ score) of the precue responsive units on each trial does not correlate with the latency to nose poke into the cue hole on that trial (supplemental Fig. S2, available at www.jneurosci.org as supplemental material). This may be because other nonattention-related processing (i.e., motor preparation and response selection) occurs during the time between cue illumination and cue hole nose poke or because our measurement of phasic changes in single-unit firing rate is not sensitive enough to correlate with behavioral latencies. Furthermore, given that rats are active and unconstrained in this task, there is considerable variability in body position and movement toward the cues between trials. This may diminish the possibility that a correlation is found between behavioral response latencies and single-unit phasic responses on each trial. Measures of large-scale neural activity, such as local field potential oscillations, may correlate better with behavioral response latency. The small population of precue responsive units (Fig. $5 B, E$ ) represented the totality of the neural response to the cue, with the rest of the population of recorded single units showing no response to the cue (supplemental Fig. S3, available at www.jneurosci.org as supplemental material). The $200 \mathrm{~ms}$ PETH bin size was used to reduce variability in precue neural activity; however, the data were also analyzed in $50 \mathrm{~ms}$ bins and results similar to those using $200 \mathrm{~ms}$ bins were found in both brain regions (supplemental Fig. S4, available at www.jneurosci.org as supplemental material).

Because the food magazine nose poke to either consume reward or end the house light extinguishment also initiated the next trial and $8 \mathrm{~s}$ intertrial interval, precue neural activity was potentially affected by reward or error processing, rather than attention. However, we found that precue activity compared between trials after food consumption and trials after errors did not differ (Fig. 6). For excitatory and inhibitory units, there was no interaction between previous reward or error and time in either the ACC $(p>0.05)$ or the mPFC $(p>0.05)$. Thus, precue phasic changes in firing represent preparatory attention.

Cue-related phasic responses were only related to whether the response choice was correct or incorrect and not to the spatial location of the cue (Fig. 7). The population activity of cue responsive units on correct trials according to which cue location (left, right, or center) was presented on each trial was not significantly 
A

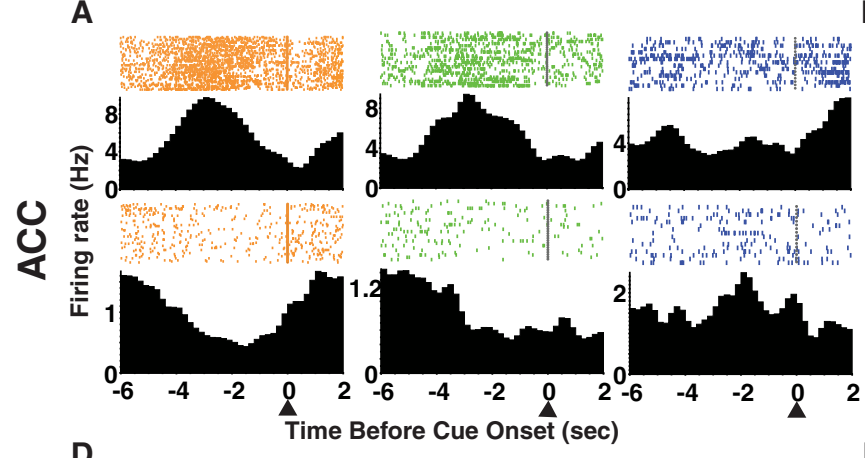

D

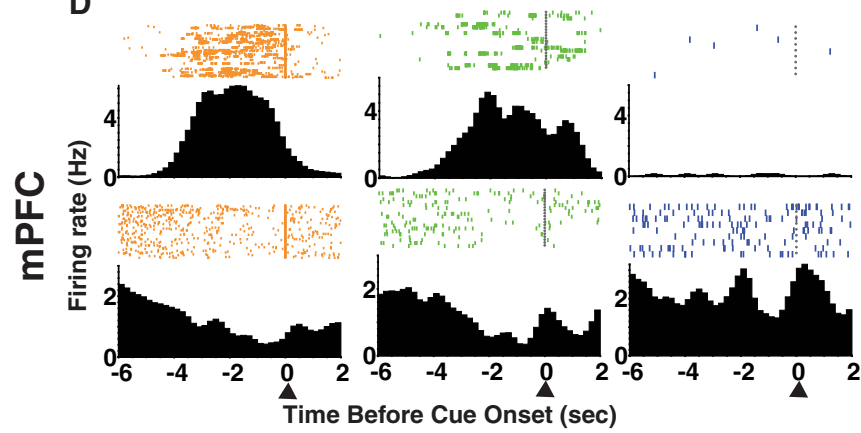

B
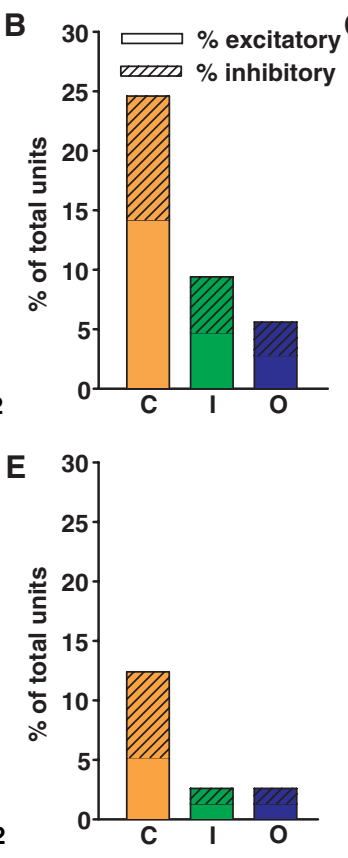

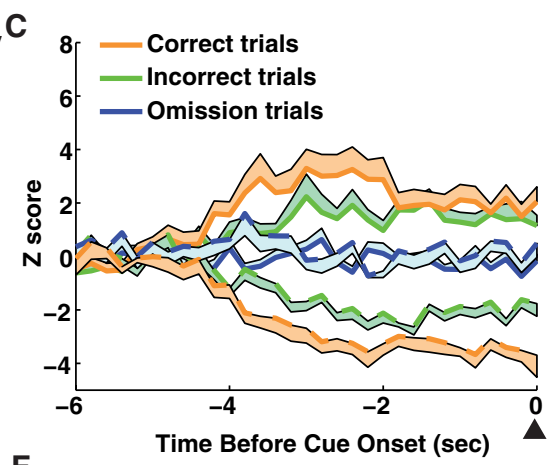

$\mathbf{F}$

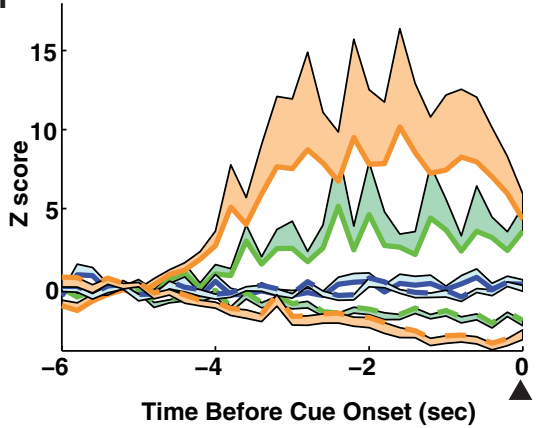

Figure 5. Precue single-unit activity. ACC ( $\boldsymbol{A}-\boldsymbol{C}$, top) and $\mathrm{mPFC}(\boldsymbol{D}-\boldsymbol{F}$, bottom). $\boldsymbol{A}$, Histograms aligned to cue onset (arrow) are shown from an example excitatory unit (top) and an example inhibitory unit (bottom). The unit's phasic response before the cue on correct trials (red) and the activity of the same unit on incorrect trials (green) and omission trials (blue). $\boldsymbol{B}$, The percentage of total units which responded before the cue on correct trials (red), incorrect trials (green), and omission trials (blue). Excitatory units are shown as solid bars, and inhibitory units are shown as hashed bars. C, The population activity (excitatory, solid line; inhibitory, dashed line) of precue responsive units on correct trials and the activity of those same units on incorrect and omission trials. The lines represent the mean activity and the shaded areas represent the SE. $\boldsymbol{D}-\boldsymbol{F}$, PFC, same as above.

Table 1. The number and percentage of units responding to behavioral events

\begin{tabular}{|c|c|c|c|c|}
\hline & \multicolumn{2}{|c|}{$\begin{array}{l}\text { Responsive units in ACC } \\
(n=106)\end{array}$} & \multicolumn{2}{|c|}{$\begin{array}{l}\text { Responsive units in mPFC } \\
(n=153)\end{array}$} \\
\hline & Increase & Decrease & Increase & Decrease \\
\hline \multicolumn{5}{|l|}{ Precue period } \\
\hline Correct trials & 16 (15.1\%) & $11(10.3 \%)$ & $8(5.2 \%)$ & $11(7.2 \%)$ \\
\hline Incorrect trials & $5(4.7 \%)$ & $5(4.7 \%)$ & $2(1.3 \%)$ & $2(1.3 \%)$ \\
\hline Omission trials & $3(2.8 \%)$ & $3(2.8 \%)$ & $2(1.3 \%)$ & $2(1.3 \%)$ \\
\hline \multicolumn{5}{|l|}{ Cue hole nose poke } \\
\hline Correct trials & $7(6.6 \%)$ & $6(5.7 \%)$ & $9(5.9 \%)$ & $6(3.9 \%)$ \\
\hline Incorrect trials & $3(3.0 \%)$ & $1(0.9 \%)$ & $7(4.6 \%)$ & $0(0 \%)$ \\
\hline Premature trials & $6(5.7 \%)$ & $0(0 \%)$ & $6(3.9 \%)$ & $0(0 \%)$ \\
\hline \multicolumn{5}{|c|}{ Food magazine nose poke } \\
\hline Food consumption & $13(12.3 \%)$ & 12 (11.3\%) & $14(9.2 \%)$ & $6(3.9 \%)$ \\
\hline Error resolution & $8(7.6 \%)$ & $5(4.7 \%)$ & $5(3.3 \%)$ & $0(0 \%)$ \\
\hline
\end{tabular}

different between cue locations for excitatory or inhibitory units in the ACC $(p>0.05)$ or the mPFC $(p>0.05)$.

\section{Phasic neural responses related to error feedback}

Significant phasic responses related to motor execution and error processing were observed in the MPFC and ACC, respectively. Phasic responses related to cue hole nose poke onset were divided into three groups: correct, incorrect, and premature nose poke types. The percentage of total units recorded that responded with excitation to the three cue hole nose poke events was similar between brain regions (Table 1). Although the number of responsive units is small, our criteria for considering a unit responsive (four consecutive time bins) are sufficiently stringent such that it is unlikely that a unit would be significant by chance. Furthermore, the response was from a distinct population of units, with the remainder of recorded units not contributing to the response (supplemental Figs. S5, S6, and S7, available at www. jneurosci.org as supplemental material, show correct, incorrect, and premature nose poke histograms, respectively). Phasic inhibitory responses were associated with correct cue hole nose pokes in both the ACC and mPFC (Fig. 8B,E). These inhibitory units remained inhibited during the entire window of analysis around the correct cue hole nose poke ( -1 to $+1 \mathrm{~s})$ and did not return to baseline (Fig. $8 C, F)$.

In the $\mathrm{mPFC}$, phasic excitations were observed for all three cue hole nose poke response types. However, the activity patterns related to each cue hole nose poke type were not significantly different from one another (nose poke type $\times$ time interaction, $p>0.05)$. Error-related activity was observed in the ACC only (Fig. 8C). The error activity consisted of a phasic excitation after the incorrect cue hole nose poke. A significant interaction between trial type and time was observed $\left(F_{(78,585)}=2.90, p<\right.$ 0.0001 ). It is important to note that a large phasic neural response after premature cue hole nose pokes, which were also errors, was not found in the ACC or mPFC. A premature cue hole nose poke is a different type of error than an incorrect cue hole nose poke in that it occurs before cue onset and results in a timeout that cannot be resolved by the rat. On incorrect trials, an incorrect action has been made to the cue that must be dealt with by nose poking into the food magazine; thus, other actions need to be planned to continue the task.

\section{Phasic neural activity related to resolving error}

In addition to the error-related activity after incorrect cue hole nose pokes observed exclusively in the ACC, there was a larger number of single units in the ACC that were responsive to resolving errors, that is, the intentional action of the rat to end the timeout and initiate the next trial. In the ACC, $12.3 \%$ of the total units recorded responded with either excitation or inhibition to 
A

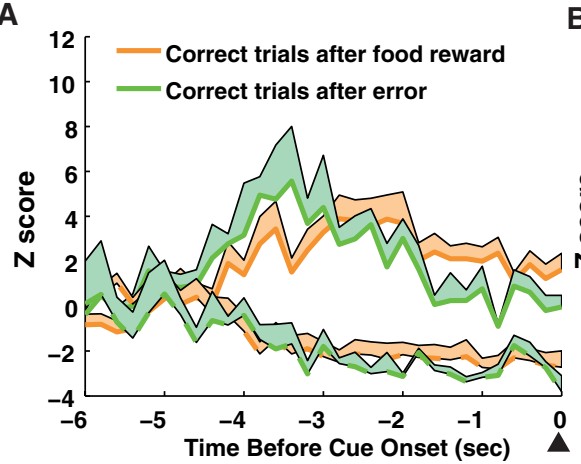

B

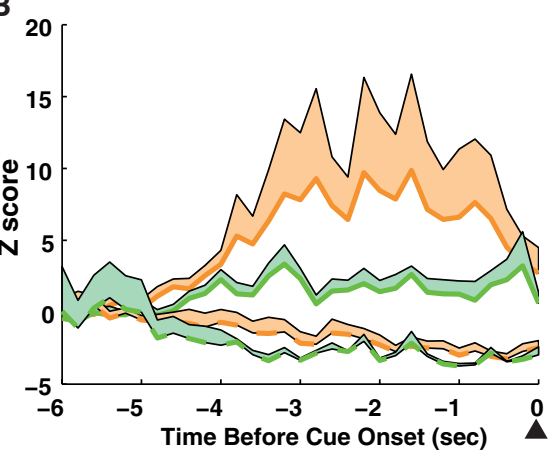

Figure 6. Activity of precue responsive units on correct trials grouped by trials after previous food rewards (orange) or trials after errors (green). The population activity (excitatory, solid line; inhibitory, dashed line) of precue responsive units in the ACC $(\boldsymbol{A})$ or $\mathrm{mPFC}(\boldsymbol{B})$. The lines represent the mean activity and the shaded areas represent the SE.

A

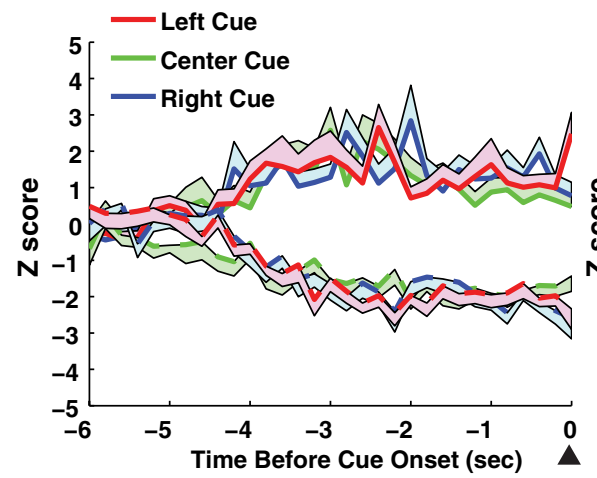

B

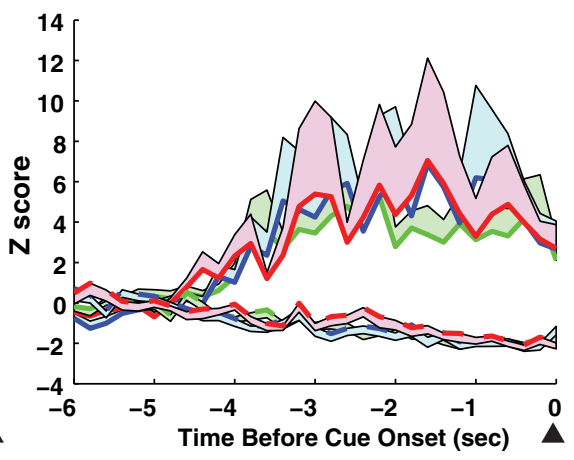

Figure 7. Precue activity for each cue location. $\mathrm{ACC}(\boldsymbol{A})$ and $\mathrm{mPFC}(\boldsymbol{B})$. Precue responsive cells were divided into three groups, depending on which of the three cue holes illuminated during that trial. No difference in excitatory or inhibitory responses was observed between cue locations (cue location $\times$ time interaction, $p>0.05$ ). The lines represent the mean activity and the shaded areas represent the SE.
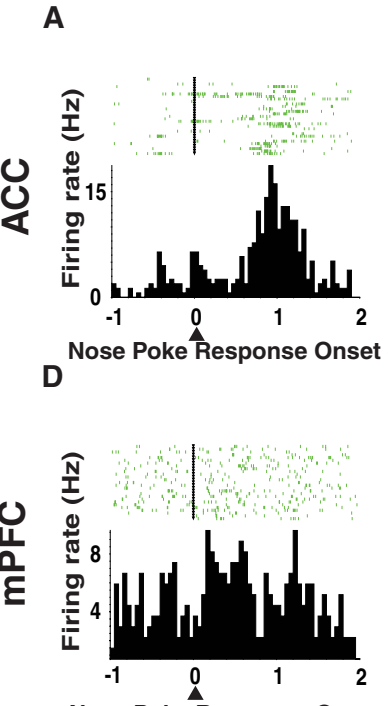

Nose Poke Response Onset (sec)
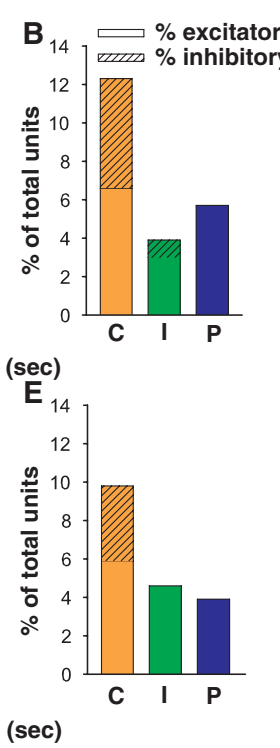

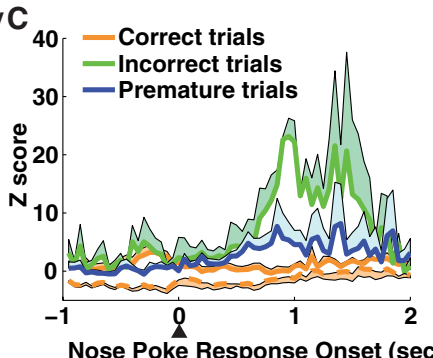

$\mathbf{F}$

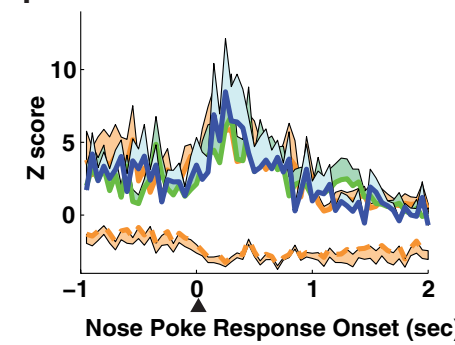

Figure 8. Cue hole nose poke-related single-unit activity. ACC ( $\boldsymbol{A}-\boldsymbol{C}$, top) and $\mathrm{mPFC}(\boldsymbol{D}-\boldsymbol{F}$, bottom). Nose pokes into the cue holes were divided into three groups: correct, incorrect, and premature (before the cue light). $\boldsymbol{A}, \boldsymbol{D}$, Single-unit examples of excitatory units that responded on incorrect cue hole nose pokes. $\boldsymbol{B}, \boldsymbol{E}$, The percentage of total units which responded during a -1 to +1 window around cue hole nose poke onset. Excitatory units are shown as solid bar and inhibitory units are shown as hashed bar. A similar proportion of units responded in each region. Correct cue hole nose pokes were associated with a significant number of inhibitory responses. $\boldsymbol{C}, \boldsymbol{F}$, The population activity (excitatory, solid line; inhibitory, dashed line) of cue hole nose pokeresponsive units. Neural activity is aligned with nose poke entry into the cue hole (arrow). The lines represent the mean activity and the shaded areas represent the SE. No difference was found between activity in the PFC (trial type $\times$ time interaction, $p>$ $0.05)$, but a phasic response to incorrect cue hole nose pokes was observed in the ACC $(p<0.01)$.

food magazine nose pokes on error trials, whereas in the mPFC, only $3.3 \%$ responded (Table 1). Furthermore, in the ACC only, the population response patterns of inhibitory units discriminated food magazine nose pokes that resolved errors from food magazine nose pokes for consuming reward (Fig. 9B). A significant interaction between food magazine nose poke type and time was observed for inhibitory units in the $\operatorname{ACC}\left(F_{(39,585)}=3.60\right.$, $p<0.0001)$. The inhibitory response related to nose poking for resolving an error returned to baseline, whereas inhibition to reward consumption remained inhibited. For excitatory units, there was no significant interaction between food magazine nose poke type (reward or ending punishment) and time in the ACC $(p>0.05)$ or the $\mathrm{mPFC}(p>0.05)$. The units responsive to food magazine nose poke events were a distinct population of units, with the nonresponsive units having no phasic response (supplemental Figs. S8, S9, available at www.jneurosci.org as supplemental material).

A larger number of single units in the ACC were also responsive to food magazine nose pokes for reward consumption. In the ACC, $23.6 \%$ of the total units recorded responded with either excitation or inhibition to food magazine nose pokes on reward consumption trials, whereas in the mPFC, only $13.1 \%$ responded (Table 1 ). Furthermore, a large phasic excitation before food magazine nose poke for reward consumption was observed only in the ACC (Fig. 9A).

\section{Discussion}

During the same behavioral sequence, error-related signals and the preparatory period were represented by the ACC. The mPFC only responded to the preparatory period. On incorrect trials, the number of responsive units and the magnitude of precue activity were reduced in both regions suggesting that these phasic responses are related to the degree of preparatory attention and subsequent correct or incorrect choice. The same relationship between precue activity and subsequent performance was found for inhibitory phasic responses in both brain regions, suggesting that phasic inhibition contributes to preparatory attention. ACC units discriminated food magazine nose pokes to retrieve reward from those which resolved error when the rat ended the timeout, suggesting that these neurons represent actionoutcome-related information and aid in resolving the error. 
Error-related signaling and outcome monitoring in the ACC

Consistent with previous studies (Miltner et al., 1997; Ruchsow et al., 2002; Ito et al., 2003; Quilodran et al., 2008), a phasic excitatory error-related response was observed after an incorrect cue hole nose poke in the ACC. This error signal could be used by the brain for conflict monitoring (Botvinick et al., 2004) or as a reinforcement learning signal (Nieuwenhuis et al., 2004), both of which are currently debated using studies which directly test those questions. Inhibitory units in the ACC discriminated between food magazine nose pokes for food reward and nose pokes to resolve error (i.e., end the timeout). Additionally, a larger percentage of units in the ACC responded to food magazine nose pokes for reward consumption and for error resolution compared with the mPFC. These data suggest that the ACC is more sensitive to outcome-related information, compared with the mPFC. Thus, the ACC may represent outcomerelated information, in addition to detecting errors.

The ACC and mPFC both participate in preparatory attention

One theory of preparatory attention predicts that the mPFC/DLPFC should activate during preparatory periods, whereas the ACC should represent errors (Botvinick et al., 2004). Our recordings in the rodent $\mathrm{mPFC}$ [which is functionally homologous to the primate $\mathrm{mPFC} / \mathrm{DLPFC}$ (Uylings et al., 2003)] are similar to the anticipatory single-unit responses that have been recorded in the monkey $\mathrm{mPFC/}$ DLPFC (Niki and Watanabe, 1979; Pragay et al., 1987). Thus, our results are consistent with a role for the mPFC/DLPFC in preparatory attention.

However, the demonstration of preparatory activity in the ACC is consistent with the view that this brain region plays a direct role in preparatory attention (Posner and DiGirolamo, 1998; Weissman et al., 2005; Roelofs et al., 2006). Precue preparatory activity has been shown previously in the monkey ACC (Niki and Watanabe, 1979; Koyama et al., 2001; Johnston et al., 2007). Furthermore, our finding of reduced preparatory activity in the ACC (and mPFC) on incorrect trials is consistent with reduced human ACC eventrelated potentials, on trials with reduced preparatory attention (Padilla et al., 2006). Thus, the ACC has a dual role in goaldirected behavior, both detecting errors and responding during preparatory periods.

It is important to note that the preparatory activity could reflect reward prediction or expectation (Shidara and Richmond, 2002), rather than selection of stimuli, per se. Thus, fluctuations in internal motivations may lead to reduced expectancy of reward and subsequent incorrect performance. However, preparatory activity on incorrect trials was reduced compared with correct

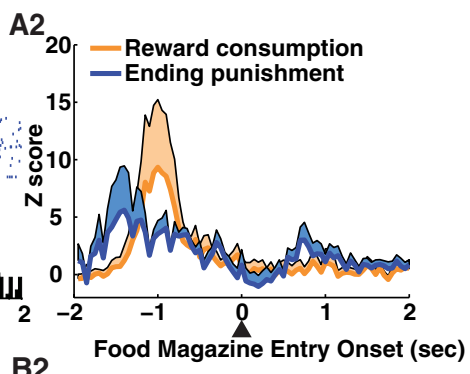

B2

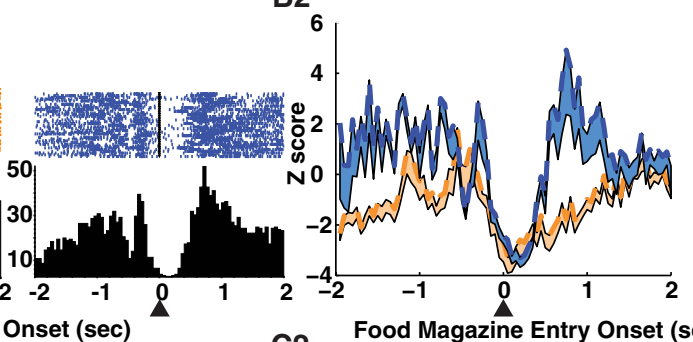

C2 10

Food Magazine Entry Onset (sec)

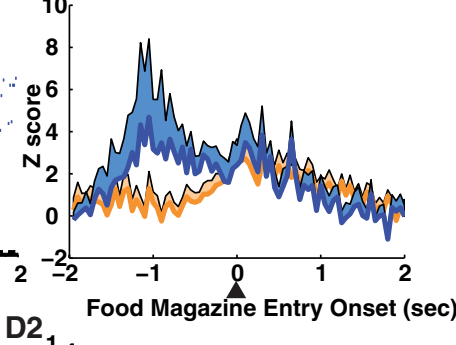

D2 1

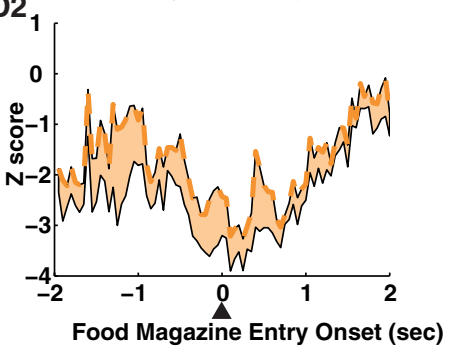

Figure 9. Reward consumption and error resolution-related single-unit activity. ACC ( $\boldsymbol{A}$, excitatory responses; $\boldsymbol{B}$, inhibitory groups: nose pokes that consumed reward and nose pokes that resolved an error. Both types of food magazine nose pokes began resolution (blue). Neural activity is shown in the same scheme for the mPFC (excitatory units: $\mathbf{C 1}, \mathbf{C}$; inhibitory units: D1, D2). The lines represent the mean activity and the shaded areas represent the SE.

trials, although the same information, or lack of information, about reward is available to the rat on both trial types. This strongly suggests that the preparatory activity reflects attention to a stimulus, rather than expectation of reward.

\section{Inhibition of ACC and PFC units during attention and} goal-directed behavior

The finding of substantial inhibition during the preparatory period and a reduction of this inhibition on incorrect trials suggests that excitation of local inhibitory interneurons and/or inhibitory afferents are brought online during attention and goal-directed behavior. Local interneurons in the PFC modulate the firing of pyramidal cells and abnormalities in interneurons are present in schizophrenia, which suggests that interneurons play a critical role in PFC neural activity patterns and in its control of cognition (Lewis et al., 2003; Ford et al., 2007). The inhibition of putative pyramidal cells during the precue period may thus be attributable 
to the excitation of local PFC interneurons via thalamic inputs (Rotaru et al., 2005). However, another source of inhibition may come from the neuromodulators dopamine and norepinephrine. Tonic dopamine and norepinephrine levels, as assessed by in vivo microdialysis, are increased during cognitive task performance (Dalley et al., 2002; Rossetti and Carboni, 2005; Stefani and Moghaddam, 2006). Both neurotransmitters have a primarily inhibitory influence on PFC pyramidal cells, with the dopamine afferents acting via $\mathrm{D}_{2}$ receptor stimulation (Mantz et al., 1988; Pirot et al., 1992). Reduced PFC dopamine and norepinephrine activity has been implicated in attentional and other cognitive deficits associated with attention- deficit/hyperactivity disorder and schizophrenia (van Kammen et al., 1986; Davis et al., 1991; Suhara et al., 2002; Abi-Dargham, 2004; Goldman-Rakic et al., 2004) and drugs that enhance PFC dopamine transmission are used to improve executive functions in patients (Arnsten and Vijayraghavan, 2006; Berridge et al., 2006; Vijayraghavan et al., 2007). In that context, it is interesting that systemic administration of a dopamine releaser, such as amphetamine, inhibits PFC pyramidal cells in awake animals (Homayoun and Moghaddam, 2006). Thus, further investigation of the neuronal mechanisms that may mediate this inhibition may be critical for delineating the role of inhibition during attention and goal-directed behavior.

\section{The ACC and mPFC: preparatory attention for different aspects of goal-directed behavior}

The preparatory attention signal in the ACC may link selection of stimuli to trial outcomes, whereas the preparatory attention from the mPFC may select stimuli for use in stimulus-response rules. Preparatory ACC unit activity is related to the degree of reward expectancy and, thus, the degree of motivation (Shidara and Richmond, 2002). Futhermore, preparatory attention is increased when motivation to select a stimulus is increased (Sarter et al., 2006). Accordingly, as the organism experiences increased motivation, its preparatory attention is also increased, as represented by precue activity. The preparatory signal in the ACC may link the selected stimulus with ACC processing of motivation and trial outcomes.

However, given the well demonstrated role of the mPFC/ DLPFC in representing stimulus-response associations (Quintana and Fuster, 1992; Asaad et al., 1998; Rainer et al., 1998; Passingham et al., 2000; Lauwereyns et al., 2001; Schoenbaum et al., 2006) and selecting stimulus-response mappings (Miller and Cohen, 2001), the preparatory activity in the mPFC may be important for linking a selected stimulus with stimulus-response rules and holding that information in working memory. Indeed, once a task is well learned, the rat mPFC is not necessary for action-outcome associations, only stimulus-response associations (Corbit and Balleine, 2003; Ostlund and Balleine, 2005). Thus, the ACC and mPFC may both participate in preparatory attention, with the former relating the trial outcome and motivation and the latter relating to stimulus-response rules.

\section{Conclusion}

We demonstrate that the ACC responds to error-related events, and along with the $\mathrm{mPFC}$ encodes preparatory attention. These results are consistent with the theory that the mPFC plays a role in preparatory attention but that the ACC plays a dual role in both preparatory attention and representing error-related events during the same task. We also propose that both ACC and mPFC regulate preparatory attention but focus that control on linking selected stimuli to trial outcomes and stimulus-response rules, respectively.

\section{References}

Abi-Dargham A (2004) Do we still believe in the dopamine hypothesis? New data bring new evidence. Int J Neuropsychopharmacol 7 [Suppl 1]:S1-S5. Arnsten AF, Vijayraghavan S (2006) Staying in touch with methylphenidate: AHDH and sensory processing. Focus on "methylphenidate enhances noradrenergic transmission and suppresses mid- and long-latency sensory responses in the primary somatosensory cortex of awake rats." J Neurophysiol 96:524-525.

Asaad WF, Rainer G, Miller EK (1998) Neural activity in the primate prefrontal cortex during associative learning. Neuron 21:1399-1407.

Berridge CW, Devilbiss DM, Andrzejewski ME, Arnsten AF, Kelley AE, Schmeichel B, Hamilton C, Spencer RC (2006) Methylphenidate preferentially increases catecholamine neurotransmission within the prefrontal cortex at low doses that enhance cognitive function. Biol Psychiatry 60:1111-1120.

Botvinick MM, Cohen JD, Carter CS (2004) Conflict monitoring and anterior cingulate cortex: an update. Trends Cogn Sci 8:539-546.

Carli M, Robbins TW, Evenden JL, Everitt BJ (1983) Effects of lesions to ascending noradrenergic neurones on performance of a 5-choice serial reaction task in rats; implications for theories of dorsal noradrenergic bundle function based on selective attention and arousal. Behav Brain Res 9:361-380.

Chudasama Y, Passetti F, Rhodes SE, Lopian D, Desai A, Robbins TW (2003) Dissociable aspects of performance on the 5-choice serial reaction time task following lesions of the dorsal anterior cingulate, infralimbic and orbitofrontal cortex in the rat: differential effects on selectivity, impulsivity and compulsivity. Behav Brain Res 146:105-119.

Corbit LH, Balleine BW (2003) The role of prelimbic cortex in instrumental conditioning. Behav Brain Res 146:145-157.

Dalley JW, Theobald DE, Pereira EA, Li PM, Robbins TW (2002) Specific abnormalities in serotonin release in the prefrontal cortex of isolationreared rats measured during behavioural performance of a task assessing visuospatial attention and impulsivity. Psychopharmacology (Berl) 164:329-340.

Davis KL, Kahn RS, Ko G, Davidson M (1991) Dopamine in schizophrenia: a review and reconceptualization. Am J Psychiatry 148:1474-1486.

Ford JM, Krystal JH, Mathalon DH (2007) Neural synchrony in schizophrenia: from networks to new treatments. Schizophr Bull 33:848-852.

Goldman-Rakic PS, Castner SA, Svensson TH, Siever LJ, Williams GV (2004) Targeting the dopamine D1 receptor in schizophrenia: insights for cognitive dysfunction. Psychopharmacology (Berl) 174:3-16.

Homayoun H, Moghaddam B (2006) Progression of cellular adaptations in medial prefrontal and orbitofrontal cortex in response to repeated amphetamine. J Neurosci 26:8025-8039.

Ito S, Stuphorn V, Brown JW, Schall JD (2003) Performance monitoring by the anterior cingulate cortex during saccade countermanding. Science 302:120-122.

Johnston K, Levin HM, Koval MJ, Everling S (2007) Top-down controlsignal dynamics in anterior cingulate and prefrontal cortex neurons following task switching. Neuron 53:453-462.

Koyama T, Kato K, Tanaka YZ, Mikami A (2001) Anterior cingulate activity during pain-avoidance and reward tasks in monkeys. Neurosci Res 39:421-430.

Lauwereyns J, Sakagami M, Tsutsui K, Kobayashi S, Koizumi M, Hikosaka O (2001) Responses to task-irrelevant visual features by primate prefrontal neurons. J Neurophysiol 86:2001-2010.

Lewis BL, O'Donnell P (2000) Ventral tegmental area afferents to the prefrontal cortex maintain membrane potential 'up' states in pyramidal neurons via $\mathrm{D}(1)$ dopamine receptors. Cereb Cortex 10:1168-1175.

Lewis DA, Glantz LA, Pierri JN, Sweet RA (2003) Altered cortical glutamate neurotransmission in schizophrenia: evidence from morphological studies of pyramidal neurons. Ann N Y Acad Sci 1003:102-112.

Mantz J, Milla C, Glowinski J, Thierry AM (1988) Differential effects of ascending neurons containing dopamine and noradrenaline in the control of spontaneous activity and of evoked responses in the rat prefrontal cortex. Neuroscience 27:517-526.

Miller EK, Cohen JD (2001) An integrative theory of prefrontal cortex function. Annu Rev Neurosci 24:167-202.

Miltner WHR, Braun CH, Coles MGH (1997) Event-related brain poten- 
tials following incorrect feedback in a time-estimation task: evidence for a "generic" neural system for error detection. J Cogn Neurosci 9:788-798.

Ng CW, Noblejas MI, Rodefer JS, Smith CB, Poremba A (2007) Double dissociation of attentional resources: prefrontal versus cingulate cortices. J Neurosci 27:12123-12131.

Nieuwenhuis S, Holroyd CB, Mol N, Coles MG (2004) Reinforcementrelated brain potentials from medial frontal cortex: origins and functional significance. Neurosci Biobehav Rev 28:441-448.

Niki H, Watanabe M (1979) Prefrontal and cingulate unit activity during timing behavior in the monkey. Brain Res 171:213-224.

Ostlund SB, Balleine BW (2005) Lesions of medial prefrontal cortex disrupt the acquisition but not the expression of goal-directed learning. J Neurosci 25:7763-7770.

Padilla ML, Wood RA, Hale LA, Knight RT (2006) Lapses in a prefrontalextrastriate preparatory attention network predict mistakes. J Cogn Neurosci 18:1477-1487.

Passetti F, Chudasama Y, Robbins TW (2002) The frontal cortex of the rat and visual attentional performance: dissociable functions of distinct medial prefrontal subregions. Cereb Cortex 12:1254-1268.

Passingham RE, Toni I, Rushworth MF (2000) Specialization within the prefrontal cortex: the ventral prefrontal cortex and associative learning. Exp Brain Res 133:103-113.

Pirot S, Godbout R, Mantz J, Tassin JP, Glowinski J, Thierry AM (1992) Inhibitory effects of ventral tegmental area stimulation on the activity of prefrontal cortical neurons: evidence for the involvement of both dopaminergic and GABAergic components. Neuroscience 49:857-865.

Posner MI, DiGirolamo GJ (1998) Executive attention: conflict, target detection, and cognitive control. In: The attentive brain (Parasuraman R, ed), pp 401-423. Cambridge, MA: MIT.

Pragay EB, Mirsky AF, Nakamura RK (1987) Attention-related unit activity in the frontal association cortex during a go/no-go visual discrimination task. Exp Neurol 96:481-500.

Quilodran R, Rothé M, Procyk E (2008) Behavioral shifts and action valuation in the anterior cingulate cortex. Neuron 57:314-325.

Quintana J, Fuster JM (1992) Mnemonic and predictive functions of cortical neurons in a memory task. Neuroreport 3:721-724.

Rainer G, Asaad WF, Miller EK (1998) Selective representation of relevant information by neurons in the primate prefrontal cortex. Nature 393:577-579.
Roelofs A, van Turennout M, Coles MG (2006) Anterior cingulate cortex activity can be independent of response conflict in Stroop-like tasks. Proc Natl Acad Sci U S A 103:13884-13889.

Rossetti ZL, Carboni S (2005) Noradrenaline and dopamine elevations in the rat prefrontal cortex in spatial working memory. J Neurosci 25:2322-2329.

Rotaru DC, Barrionuevo G, Sesack SR (2005) Mediodorsal thalamic afferents to layer III of the rat prefrontal cortex: synaptic relationships to subclasses of interneurons. J Comp Neurol 490:220-238.

Ruchsow M, Grothe J, Spitzer M, Kiefer M (2002) Human anterior cingulate cortex is activated by negative feedback: evidence from event-related potentials in a guessing task. Neurosci Lett 325:203-206.

Sarter M, Gehring WJ, Kozak R (2006) More attention must be paid: the neurobiology of attentional effort. Brain Res Rev 51:145-160.

Schoenbaum G, Roesch MR, Stalnaker TA (2006) Orbitofrontal cortex, decision-making and drug addiction. Trends Neurosci 29:116-124.

Shidara M, Richmond BJ (2002) Anterior cingulate: single neuronal signals related to degree of reward expectancy. Science 296:1709-1711.

Stefani MR, Moghaddam B (2002) Rule learning and reward contingency are associated with dissociable patterns of dopamine activation in the rat perfrontal cortex, nucleus accumbens, and dorsal striatum. J Neurosci 26:8810-8818.

Suhara T, Okubo Y, Yasuno F, Sudo Y, Inoue M, Ichimiya T, Nakashima Y, Nakayama K, Tanada S, Suzuki K, Halldin C, Farde L (2002) Decreased dopamine $\mathrm{D} 2$ receptor binding in the anterior cingulate cortex in schizophrenia. Arch Gen Psychiatry 59:25-30.

Uylings HB, Groenewegen HJ, Kolb B (2003) Do rats have a prefrontal cortex? Behav Brain Res 146:3-17.

van Kammen DP, van Kammen WB, Mann LS, Seppala T, Linnoila M (1986) Dopamine metabolism in the cerebrospinal fluid of drug-free schizophrenic patients with and without cortical atrophy. Arch Gen Psychiatry 43:978-983.

Vijayraghavan S, Wang M, Birnbaum SG, Williams GV, Arnsten AF (2007) Inverted-U dopamine $\mathrm{D} 1$ receptor actions on prefrontal neurons engaged in working memory. Nat Neurosci 10:376-384.

Weissman DH, Gopalakrishnan A, Hazlett CJ, Woldorff MG (2005) Dorsal anterior cingulate cortex resolves conflict from distracting stimuli by boosting attention toward relevant events. Cereb Cortex 15:229-237. 